\title{
Deletion of the ubiquitin-conjugating enzyme Ubc2 confers resistance to methylmercury in budding yeast by promoting Whi2 degradation
}

\author{
Gi-Wook Hwang'1, Fujio Mastuyama'1, Tsutomu Takahashi', Jin-Yong Lee ${ }^{1,2}$ \\ and Akira Naganuma ${ }^{1}$ \\ ${ }^{1}$ Laboratory of Molecular and Biochemical Toxicology, Graduate School of Pharmaceutical Sciences, \\ Tohoku University, Aoba-ku, Sendai 980-8578, Japan \\ ${ }^{2}$ Laboratory of Pharmaceutical Health Sciences, School of Pharmacy, Aichi Gakuin University, \\ 1-100 Kusumoto-cho, Chikusa-ku, Nagoya 464-8650, Japan
}

(Received September 25, 2012; Accepted March 4, 2013)

\begin{abstract}
Ubiquitin-conjugating enzymes involved in sensitivity to methylmercury in yeast were identified by deletion analysis, which showed that Ubc2- or Ubp13-deficiency conferred resistance to methylmercury. Whi2, which was previously shown to be associated with increased methylmercury toxicity and is intracellularly degraded via the ubiquitin-proteasome system, was expressed at significantly lower levels in Ubc2-deficient yeast than in wild-type yeast. Ubc2/Whi2 double-deficient yeast showed neither an additive nor synergistic increase in methylmercury resistance. Our results indicate that Ubc2 may increase the sensitivity to methylmercury in yeast by inhibiting the proteasomal degradation of Whi2.
\end{abstract}

Key words: Methylmercury, Toxicity, Ubiquitin-conjugating enzyme, Whi2, Yeast

\section{INTRODUCTION}

Methylmercury is a pollutant widely known as the causative agent of Minamata disease (Hwang et al., 2011; Sanfeliu et al., 2003; Watanabe et al., 2012; YaginumaSakurai et al., 2012; Yoshida et al., 2011). The ubiquitinproteasome system, which functions as a selective protein degradation system, plays a role in methylmercury toxicity (Hwang et al., 2002, 2005, 2006, 2009 and 2012a). Protein ubiquitination occurs through a series of steps catalyzed by E1ubiquitin-activating enzymes, E2 ubiquitin conjugating enzymes, and E3 ubiquitin ligases (Pickart, 2001). Polyubiquitin chains formed by these enzymes are involved in signal transduction and protein transport, and target proteins for proteasomal degradation (Pickart and Fushman, 2004; Sun and Chen, 2004). Overexpression of Cdc34, a ubiquitin-conjugating enzyme, confers resistance to methylmercury in yeast by enhancing the ubiquitination and subsequent proteasomal degradation of Whi2, a protein that increases methylmercury sensitivity in yeast (Hwang et al., 2012b). Another study showed that overexpression of $\mathrm{Ubc} 4$ or $\mathrm{Ubc} 7$, as well as $\mathrm{Cdc} 34$, confers resistance to methylmercury in yeast (Furuchi et al.,
2002). However, the involvement of other ubiquitin-conjugating enzymes in methylmercury toxicity is unknown. In the present study, we performed a deletion analysis to identify ubiquitin-conjugating enzymes involved in the sensitivity to methylmercury in yeast.

\section{MATERIALS AND METHODS}

\section{Yeast strains and media}

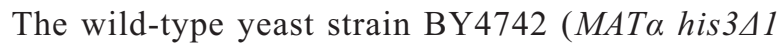
leu $2 \Delta 0$ lys $2 \Delta 0$ ura3 $\Delta 0$ ) and deletion strains in the same genetic background were purchased from Euroscarf (Frankfurt, Germany). Yeast cells were grown in yeast extract-peptone-dextrose (YPD) medium or in synthetic dextrose (SD) medium at $30^{\circ} \mathrm{C}$. The URA3-based plasmid pKT10-WHI2-HA was introduced into yeast cells using the lithium acetate procedure (Takahashi et al., 2011).

\section{Quantification of methylmercury toxicity in yeast cells}

For the spot test analysis, yeast cells $\left(10^{6}\right.$ cells $\left./ \mathrm{ml}\right)$ were grown in SD liquid medium containing methylmercuric chloride at various concentrations. After incuba- 
tion for $3 \mathrm{hr}$, cells were centrifuged and each pellet was suspended in $0.1 \mathrm{ml}$ sterilized water. An aliquot $(5 \mu \mathrm{l})$ of each suspension was spotted onto agar-solidified SD medium and incubated for $20-24 \mathrm{hr}$ at $30^{\circ} \mathrm{C}$.

\section{Immunoblotting of Whi2-HA}

Yeast-cell lysates were prepared, and immunoblotting was performed as described elsewhere. Yeast cells $\left(5 \times 10^{6}\right.$ cells $\left./ \mathrm{ml}\right)$ were grown in $10 \mathrm{ml}$ of SD (-Ura) liquid medium for $3 \mathrm{hr}$ and harvested. Approximately $0.1 \mathrm{ml}$ of cell pellet was fixed in 10\% trichloroacetic acid on ice for $30 \mathrm{~min}$ and then disrupted with glass beads in TE [100 mM Tris- $\mathrm{HCl}$ (pH 9.5) and $10 \mathrm{mM}$ EDTA] and solubilized with lysis buffer containing 1\% SDS. Yeastcell lysates were separated by SDS-polyacrylamide gel $(12.5 \%)$ electrophoresis, and proteins were transferred to an Immobilon-P membrane (Millipore Corporation, Billerica, MA, USA) and subjected to immunostaining with anti-HA (Roche, Indianapolis, IN, USA) or antiactin (Santa Cruz Biotechnology, Santa Cruz, CA, USA) antibodies.

\section{RESULTS AND DISCUSSION}

Of 13 known ubiquitin-conjugating enzymes, Cdc34 and Ubc9 are essential for yeast growth. Therefore, we examined the methylmercury sensitivity of 11 ubiquitinconjugating enzyme-deficient yeast strains and showed that yeast cells deficient in Ubc13 or Ubc2 were more sensitive to methylmercury than wild-type yeast (Fig. 1). Yeast strains deficient in other ubiquitin-conjugating

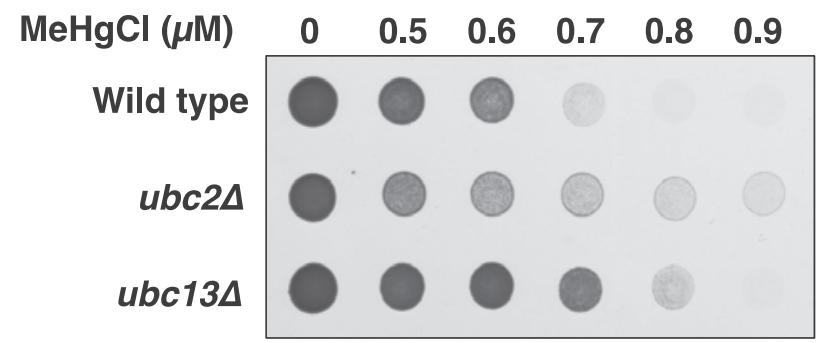

Fig. 1. Effects of Ubc2 or the Ubc13 deletion on the sensitivity of yeast cells to methylmercury. Yeast cells were grown in SD liquid medium containing different concentrations of methylmercuric chloride for $3 \mathrm{hr}$ at $30^{\circ} \mathrm{C}$. After incubation, each pellet was suspended in $0.1 \mathrm{ml}$ of sterilized water, and an aliquot $(5 \mu \mathrm{l})$ of each suspension was spotted onto agar-solidified SD medium. Plates were photographed after incubation for 20 $24 \mathrm{hr}$ at $30^{\circ} \mathrm{C}$. enzymes did not show significant differences in methylmercury sensitivity from that of the wild type (data not shown). We previously reported that overexpression of Cdc34, Ubc4 or Ubc7 confers resistance to methylmercury in yeast (Furuchi et al., 2002). In the present study, Ubc4 or Ubc7 deficiency did not significantly affect the methylmercury sensitivity of yeast, suggesting that other ubiquitin-conjugating enzymes compensate for Ubc4 or Ubc7 deficiency.

Ubc2 and Ubc13 are involved in the formation of lysine 63 (K63) linked polyubiquitin chains, and play a role in DNA repair and replication by catalyzing the K63linked ubiquitination of the proliferating cell nuclear antigen (PCNA) (Hoege et al., 2002; Stelter and Ulrich, 2003; Ulrich and Jentsch, 2000). Lysine 48 (K48) linked polyubiquitin chains function as a signal for the proteasomal degradation of proteins, whereas the attachment of K63 linked polyubiquitin chains mediated by Ubc 2 and Ubc13 does not target proteins for degradation (Pickart and Fushman, 2004; Sun and Chen, 2004). Overexpression of Cdc34 promotes the proteasomal degradation of ubiquitinated Whi 2 and therefore plays a role in acquired resistance to methylmercury (Hwang et al., 2012b). However, K63 ubiquitination mediated by Ubc2 and Ubc13 may affect the proteasomal degradation of Whi2. Therefore, we examined the effects of Ubc2 or Ubc13 deficiency on the intracellular level of Whi2, and showed that Whi2 levels were significantly lower in Ubc2-deficient yeast than in wild-type yeast, but not in Ubc13-deficient yeast (Fig. 2A). This result suggested that the Ubc2-mediated K63-linked ubiquitination of Whi2 inhibits its proteasomal degradation. To clarify the involvement of Ubc2 and Whi2 in methylmercury toxicity, we examined the sensitivity of Ubc2/Whi2 double-deficient yeast to methylmercury, which showed that deficiency in both Ubc2 and Whi2 did not result in an additive or synergistic increase in methylmercury resistance (Fig. 2B). This suggests that Ubc2 and Whi2 are involved in the development of methylmercury toxicity through the same pathway, probably via a decrease in the intracellular level of Whi2 mediated by Ubc 2 deficiency. This conjecture is supported by the observation that treatment with a proteasome inhibitor decreased the methylmercury resistance of Ubc2-deficient yeast (data not shown). Ubc2 may promote the K63linked ubiquitination of Whi2, thereby suppressing its proteasomal degradation and increasing the methylmercury sensitivity of yeast. The association between methylmercury toxicity and Ubc2- or Cdc34-mediated Whi2 degradation should be investigated further to elucidate the role of ubiquitin-conjugating enzymes in the development of methylmercury toxicity. 
Deletion of Ubc2 confers methylmercury resistance in yeast

A

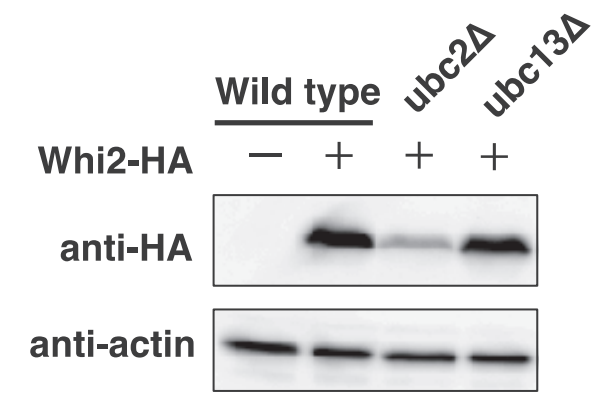

B

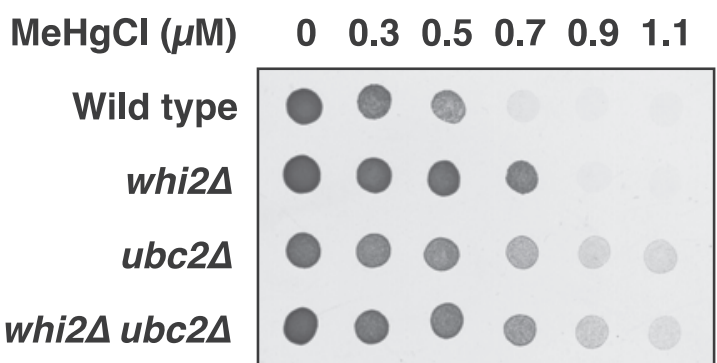

Fig. 2. Relationship between Ubc2 and Whi2 and methylmercury toxicity. (A) Effect of $U B C 2$ or the $U B C 13$ deletion on the levels of Whi2-HA. Wild type, $u b c 2 \Delta$, and $u b c 13 \Delta$ harboring Whi2-HA yeast cells were grown in SD liquid medium. Cell extracts were analyzed by immunoblotting with anti-HA antibody and anti-actin antibody as a loading control. (B) Effect of a double deletion of Ubc2 and Whi2 on the sensitivity of yeast cells to methylmercury. For details see legend to Fig. 1.

\section{REFERENCES}

Furuchi, T., Hwang, G.W. and Naganuma, A. (2002): Overexpression of the ubiquitin-conjugating enzyme Cdc34 confers resistance to methylmercury in Saccharomyces cerevisiae. Mol. Pharmacol., 61, 738-741.

Hoege, C., Pfander, B., Moldovan, G.L., Pyrowolakis, G. and Jentsch, S. (2002): RAD6-dependent DNA repair is linked to modification of PCNA by ubiquitin and SUMO. Nature, 419, 135-141.

Hwang, G.W., Furuchi, T. and Naganuma, A. (2002): A ubiquitinproteasome system is responsible for the protection of yeast and human cells against methylmercury. FASEB J., 16, 709-711.

Hwang, G.W., Ishida, Y. and Naganuma, A. (2006): Identification of
F-box proteins that are involved in resistance to methylmercury in Saccharomyces cerevisiae. FEBS Lett., 580, 6813-6818.

Hwang, G.W., Kimura, Y., Takahashi, T., Lee, J.Y. and Naganuma, A. (2012a): Identification of deubiquitinating enzymes involved in methylmercury toxicity in Saccharomyces cerevisiae. J. Toxicol. Sci., 37, 1287-1290.

Hwang, G.W., Lee, J.Y., Ryoke, K., Matsuyama, F., Kim, J.M., Takahashi, T. and Naganuma, A. (2011): Gene expression profiling using DNA microarray analysis of the cerebellum of mice treated with methylmercury. J. Toxicol. Sci., 36, 389-391.

Hwang, G.W., Ogiwara, Y., Takahashi, T. and Naganuma, A. (2012b): Ubiquitin-conjugating enzyme Cdc34 mediates methylmercury resistance in Saccharomyces cerevisiae by increasing Whi2 degradation. J. Toxicol. Sci., 37, 1283-1286.

Hwang, G.W., Sasaki, D. and Naganuma, A. (2005): Overexpression of Rad 23 confers resistance to methylmercury in Saccharomyces cerevisiae via inhibition of the degradation of ubiquitinated proteins. Mol. Pharmacol., 68, 1074-1078.

Hwang, G.W., Wada, N., Kuge, S. and Naganuma, A. (2009): Overexpression of the novel F-box protein Ymr258c confers resistance to methylmercury in Saccharomyces cerevisiae. J. Toxicol. Sci., 34, 413-416.

Pickart, C.M. (2001): Ubiquitin enters the new millennium. Mol. Cell, 8, 499-504.

Pickart, C.M. and Fushman, D. (2004): Polyubiquitin chains: polymeric protein signals. Curr. Opin. Chem. Biol., 8, 610-616.

Sanfeliu, C., Sebastià, J., Cristòfol, R. and Rodríguez-Farré, E. (2003): Neurotoxicity of organomercurial compounds. Neurotox. Res., 5, 283-305.

Stelter, P. and Ulrich, H.D. (2003): Control of spontaneous and damage-induced mutagenesis by SUMO and ubiquitin conjugation. Nature, 425, 188-191.

Sun, L. and Chen, Z.J. (2004): The novel functions of ubiquitination in signaling. Curr. Opin. Cell. Biol., 16, 119-126.

Takahashi, T., Nakashima, S., Masuda, T., Yoneda, S., Hwang, G.W. and Naganuma, A. (2011): Overexpression of CLN1, CLN2, or ERG13 increases resistance to adriamycin in Saccharomyces cerevisiae. J. Toxicol. Sci., 36, 855-857.

Ulrich, H.D. and Jentsch, S. (2000): Two RING finger proteins mediate cooperation between ubiquitin-conjugating enzymes in DNA repair. EMBO J., 19, 3388-3397.

Watanabe, N., Tayama, M., Inouye, M. and Yasutake, A. (2012): Distribution and chemical form of mercury in commercial fish tissues. J. Toxicol. Sci., 37, 853-861.

Yaginuma-Sakurai, K., Murata, K., Iwai-Shimada, M., Nakai, K., Kurokawa, N., Tatsuta, N. and Satoh, H. (2012): Hair-to-blood ratio and biological half-life of mercury: experimental study of methylmercury exposure through fish consumption in humans. J. Toxicol. Sci., 37, 123-130.

Yoshida, M., Suzuki, M., Satoh, M., Yasutake, A. and Watanabe, C. (2011): Neurobehavioral effects of combined prenatal exposure to low-level mercury vapor and methylmercury. J. Toxicol. Sci., 36, 73-80. 